\title{
The Role of p16 and MDM2 Gene Polymorphisms in
}

\author{
Seda Turgut', Muzaffer IIhan², Saime Turan³, Ozcan Karaman ${ }^{4}$, Ilhan Yaylim³, Ozlem Kucukhuseyin ${ }^{3}$, Ertugrul Tasan 4 \\ ${ }^{1}$ Bezmialem Vakif University, Department of Internal Medicine, Istanbul, Turkey \\ 2 Umraniye Training and Research Hospital, Department of Endocrinology and Metabolism, Istanbul, Turkey \\ ${ }^{3}$ Istanbul Universtiy, The Institute of Experimental Medicine, Department of Molecular Medicine, İstanbul, Turkey \\ ${ }^{4}$ Bezmialem Vakif University, Department of Endocrinology and Metabolism, Istanbul, Turkey
}

Prolactinoma is the most frequent type of functional pituitary tumors with an estimated prevalence of approximately 45 cases per 100000 population in adults (1). The vast majority of prolactinomas are benign but may lead significant morbidity associated with excessive hormone production and/or symptoms of mass effect (2). Prolactinomas are thought to arise from the proliferation of a mutated pituitary stem cell which is subjected to the growth stimuli of several permissive factors, although the pathogenetic mechanisms underlying the tumorigenesis still remain unclear $(3,4)$. Because of the limited knowledge regarding the pathogenesis, recent studies focused on the several molecular events in the cell cycle progression, cell survival, and protein synthesis in prolactinoma $(5,6)$. The present study aimed to investigate the role of $p 16(540 \mathrm{C} \rightarrow \mathrm{G}$ and $580 \mathrm{C} \rightarrow \mathrm{T})$ and $\mathrm{MDM} 2$ $(\mathrm{SNP} 309 \mathrm{~T} \rightarrow \mathrm{G})$ gene polymorphisms in tumorigenesis and characteristics of prolactinoma.

This prospective case-control study was performed in Department of Endocrinology and Metabolism, Bezmialem Vakif University Hospital, Istanbul, Turkey between 2013 and 2014. Seventy-four patients with newly diagnosed prolactinoma (59 female and 15 male) and age- and gender-matched 100 healthy subjects (78 female and 22 male) were enrolled in this study. Tumors were stratified according to the longest dimension as follows: microadenoma $(<1 \mathrm{~cm})$, macroadenoma $(\geq 1 \mathrm{~cm})$. To follow up the disease progression, clinical and laboratory examinations repeated every 3 months and MRI was performed every 6 months. Serum prolactin levels were measured by enzyme linked immunosorbent assay. DNA was extracted from peripheral blood samples then p16 and MDM2 polymorphisms were determined by polymerase chain reaction-restriction fragment polymorphism and agarose gel electrophoresis.

p16 $540 \mathrm{C} \rightarrow \mathrm{G}$ genotype distribution was found as CC: $66.2 \%$, CG: $28.4 \%$, GG: $5.4 \%$; p16 $580 \mathrm{C} \rightarrow$ T genotype distribution was found as CC: $82.4 \%$, CT: $17.6 \%$, TT: $0 \%$ and MDM2 genotype distribution was found as TT: $31.1 \%$, TG: $47.3 \%$, GG: $21.6 \%$ in patients with prolactinoma. Tumor diameter before treatment was correlated with prolactin levels before treatment and percentage of prolactin decrease with treatment $(p<0.001 r=0.719, p=0.034 r=0.256$, respectively). Tumor diameter after treatment was correlated with prolactin levels $(p<0.001 r=0.569)$. CC genotype (homozygote wild type) frequency is higher without statistical significance in both $\mathrm{p} 16540 \mathrm{C} \rightarrow \mathrm{G}$ and $\mathrm{p} 16580 \mathrm{C} \rightarrow \mathrm{T}$ polymorphisms among prolactinoma patients. The number of patients with tumor size decreased more than $50 \%$ in homozygous genotype $(T T+G G)$ carriers of MDM2 SNP309T $\rightarrow G$ is significantly higher than in heterozygous genotype (TG) carriers $(p=0.003$, OR: $0.18, \mathrm{Cl}: 0.06-0.58)$. However, other than that, no significant association was found between characteristics of prolactinoma and p16, MDM2 gene polymorphisms and alleles.

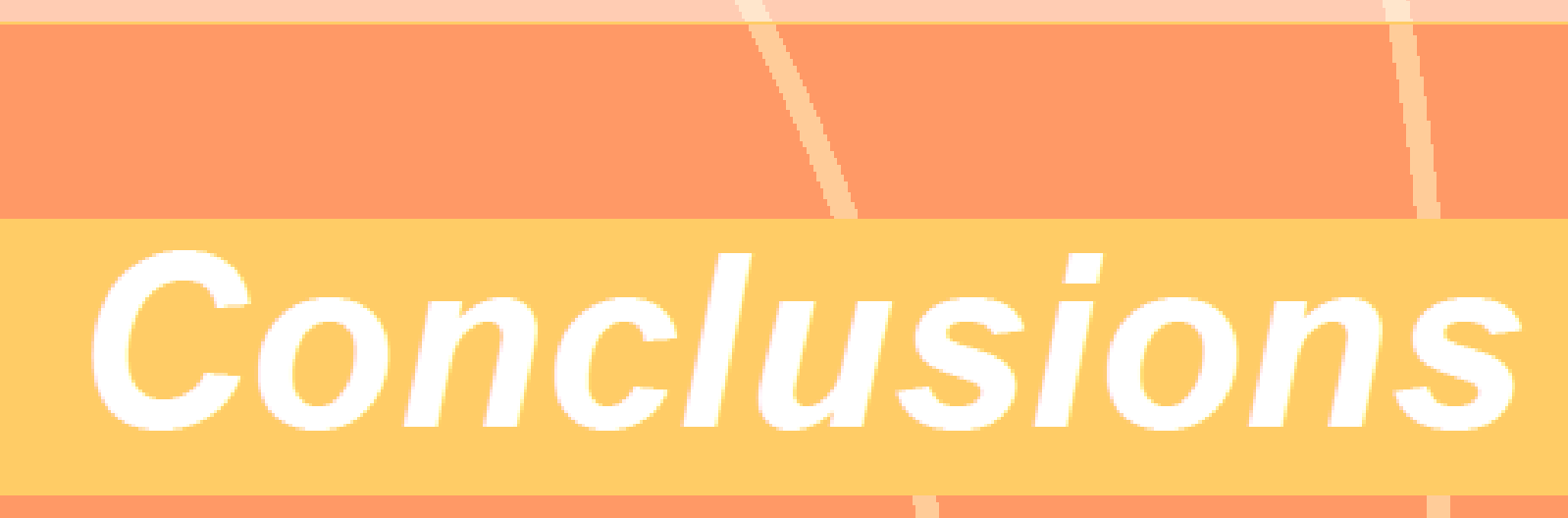

This study showed that $\mathrm{p} 16$ and MDM2 polymorphisms do not play an important role in tumorigenesis, but some genotypes of these polymorphisms could be associated with follow-up characteristics of prolactinoma. Further studies are needed to clarify the underlying mechanisms of the tumorigenesis in order to define prognostic factors and facilitate appropriate treatment choices for patients with prolactinoma.

1. Fernandez A, Karavitaki N, Wass JA. Prevalence of pituitary adenomas: a community-based, cross-sectional study in Banbury (Oxfordshire, UK). Clinical endocrinology. 2010;72(3):377-82. 2. Colao A. Pituitary tumours: the prolactinoma. Best practice \& research Clinical endocrinology \& metabolism. 2009;23(5):575-96.

3. Melmed S. Mechanisms for pituitary tumorigenesis: the plastic pituitary. The Journal of clinical investigation. 2003;112(11):1603-18.
4. Dworakowska D, Grossman AB. The pathophysiology of pittuitary adenomas. Best practice \& research Clinical endocrinology \& metabolism. 2009;23(5):525-41.

5. Tong Y, Zheng Y, Zhou J, Oyesiku NM, Koeffler HP, Melmed S. Genomic characterization of human and rat prolactinomas. Endocrinology. 2012;153(8):3679-91.
6 . Zhao L, Lin M, Wang S. Identification of human prolactinoma related genes by DNA microarray. Journal of cancer research and therapeutics. 2014;10(3):544-8. 